\title{
Simulation of Structure Dynamic Stress under Multi-load Condition in Resonance State
}

\author{
Fansong $\mathrm{Li}^{*}$, Pingbo $\mathrm{Wu}$, and Chaotao Liu \\ Traction Power State Key Laboratory, Southwest Jiaotong University, 610031 Chengdu, China
}

\begin{abstract}
Some metro vehicle lifeguard structure cracking emerged during operation, and the reason is due to resonance. In order to simulate the dynamic stress accurately in resonance state, the formula for calculating the dynamic stress under multi-load condition was deduced based on structural dynamics mechanics. During the dynamic stress simulation, firstly, the frequency response function (FRF) of each external load was calculated, which including the phase information. Then, the method was based on Fast Fourier Transform (FFT) to get the frequency domain with real and imaginary part of each external load rather than power spectrum density (PSD) calculation, avoiding loss of the phase information due to PSD method. Finally, the dynamic stress frequency domain data could be calculated by FFT results and FRFs. Based on above, the dynamic stress time history could be acquired by Inverse FFT (IFFT) of stress frequency domain data, and the stress simulation method was verified by the line test result. Compared with the conventional stress PSD calculation method, the method in this paper can retain the phase information of the each external load. Meanwhile, the conventional stress simulation method based on PSD could not get satisfactory result.
\end{abstract}

\section{Introduce}

In dynamic theory, when the frequency of external load on structure is close to the structural mode frequency, and the external load direction is the same with the mode direction at the external load point, it will cause structural resonance easily, which may lead to fracture. Due to the fatigue test cost is high, and it could not be carried out before the structure prototype finished, as a result, the numerical simulation of the dynamic stress level under working condition becomes a necessary tool during the design stage. In general, the structure resonance cracking is mainly due to that the main frequency of external load in the working environment is unknown during structure design stage, but how to accurately simulate the dynamic stress is also a problem when we get the external excitation, only the accurate dynamic stress is obtained can we predict the fatigue life. Therefore, it is very important to accurately and quickly simulate the dynamic stress of structure under the resonance state, which could be used to guide the structure optimization.

There are two common methods in structural dynamic stress simulation [1], one is quasi-static superposition method, and the other is the modal superposition method. Quasi-static superposition method [2] is suitable when the external load frequency is much lower than the structural first elastic modal frequency, but this method is not suitable for dynamic stress prediction when structure is under resonance condition [1]. In modal superposition method, the external load frequency and amplitude is the system input, and the dynamic stress is predicted according to structure inherent properties. It is a suitable method for vibration and stress prediction of structure under resonance state. However, in present study [3, 4], the input loads are often processed as PSD, which ignoring the phase information of the load in the frequency domain. When the input loadings are multiple, the phase between the loadings could not be separated. So, it could not simulate the structure dynamic stress factually under multi-load input condition.

In this paper, the simulation method of structural dynamic stress under multi-load input condition was studied, and the phase relationship between the input loadings was considered.

\section{Background}

\subsection{Research background}

As shown in Fig. 1, lifeguard structure cracking emerged in metro vehicles. And the cracking reason was found by line test, which is that the first two directional bending mode of lifeguard structure was aroused by the wheel excitation from ninth-order wheel polygon. And the cracking event is a typical vibration fatigue problem. During the line test, the different directional acceleration of lifeguard fixed end and the strain (Fig. 2) at the crack were recorded. In order to redesign a new lifeguard structure, we should know how to calculate the dynamic stress accurately according to external load in resonance

Corresponding author:lifansong2013@163.com 
state. Then the new designed structure could be verified whether satisfy the design life by the test environment load.

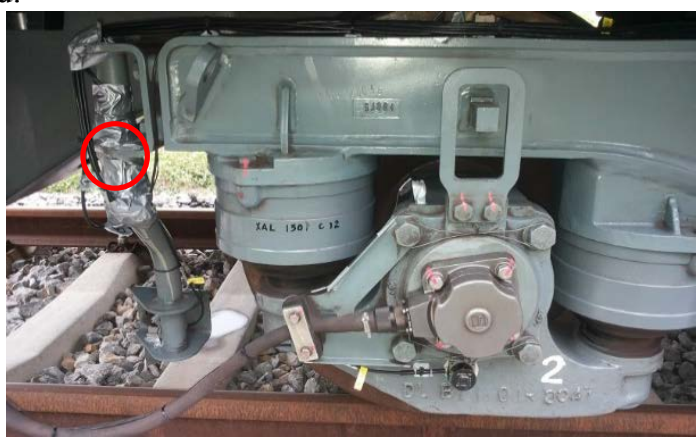

(a) The position of lifeguard of metro vehicle

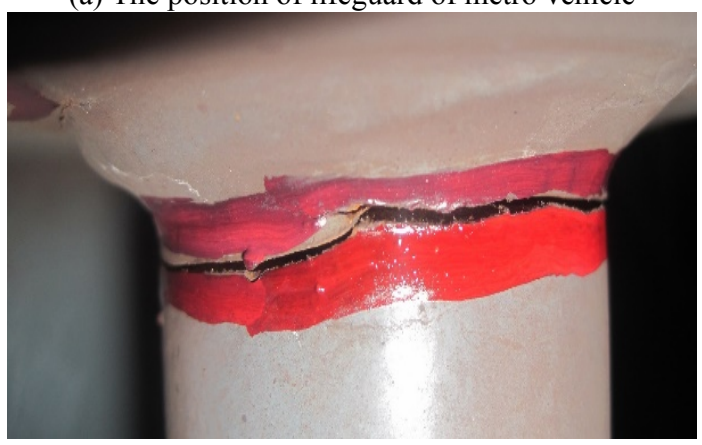

(b) Crack of lifeguard structure

Fig. 1. Lifeguard structure cracking



Fig. 2. Line test of strain of lifeguard structure

\subsection{Theoretical background}

The motion equation of structure could be given in Equation (1) [4].

$$
[\mathbf{M}]\{x\}+[\mathbf{C}]\{x\}+[\mathbf{K}]\{x\}=\{\mathbf{f}\}
$$

Where $[\mathrm{M}]$ is the mass matrix; [C] is the damping matrix; $[\mathrm{K}]$ is the stiffness matrix; $\{\mathrm{X}\}$ is the vector of degrees of freedom and $\{\mathrm{f}\}$ is the excitation force vector. Furthermore, through modal analysis the FRFs from one point on the structure to the other can be deduced.

According to the dynamic theory, the load-stress FRFs could be written in Equation (2) [5].

$$
\mathrm{H}(\omega)=\mathrm{E} \cdot \sum_{r=1}^{R} \frac{\left\{\psi_{r}^{\varepsilon}\right\}\left\{\varphi_{r}\right\}^{T}}{k_{r}-\omega^{2} m_{r}+j \omega c_{r}}
$$

Where $m_{r}$ is the $r$ th modal mass, $k_{r}$ is the $r$ th modal stiffness, $c_{r}$ is the rth modal damping, $\omega$ is the frequency of excitation. $\left\{\varphi_{r}\right\}$ is the $r$ th real value mode vector, $R$ is the total number of modes considered, $\left\{\psi_{r}^{\varepsilon}\right\}$ is called the $r$ th strain mode corresponding to $\left\{\varphi_{r}\right\}, j$ is $\sqrt{-1}, \mathrm{E}$ is material elastic module, $\omega=2 \pi f, f$ is frequency.

According to [6], when the external load is single, the stress PSD G $(f)$ can be found very rapidly by multiplying the load PSD $\mathrm{W}(f)$ and FRF $\mathrm{H}(f)$, which is shown in Equation (3).

$$
\mathrm{G}(f)=|\mathrm{H}(f)|^{2} \cdot \mathrm{W}(f)
$$

When the external load is not single but multiple, for a general circumstances with $n$ simultaneous loadings, the stress PSD G(f) can be determined by Equation (4).

$$
G(f)=\sum_{a=1}^{n} \sum_{b=1}^{n} \mathrm{H}_{a}(f) \cdot \mathrm{H}_{b}(f)^{*} \cdot \mathrm{W}_{a b}(f)
$$

Where $\mathrm{H}_{a}(f)$ and $\mathrm{H}_{b}(f)$ are FRFs relating to different two loadings, and $a$ and $b$ could be equal. $\mathrm{W}_{a b}(f)$ is the cross power spectrum density (CPSD) of load $a$ and load $b$.

\section{The FFT stress simulation method}

However, the PSDs alone do not provide information on the phase relations between diferent loadings. So, the method above of calculating stress will cause something wrong under multi-load condition. In order to overcome the problem, this paper suggest to use FFT method rather than PSD method. From Equation (2) we know the FRF was complex, which including phase relations between input and output (load and stress), so if FFT of external loadings is adopted, the phase information of loading itself will be retained in frequency domain, which could known in Equation (5). In the case of multi-load input, that is, when the number of external loadings is more than one, assuming that the number is $k$. The $i$ th external loading FFT could be described as:

$$
\mathrm{F}_{i}(\omega)=\sum_{m=0}^{N-1} f_{i}(n) \cos m \omega-j \sum_{m=0}^{N-1} f_{i}(n) \sin m \omega
$$

Where $N$ is the sampling length of load.

Then, the frequency domain of stress could be calculated by Equation (6) based on dynamic mechanics.

$$
\sigma(\omega)=\sum_{i=1}^{k} F_{i}(\omega) \cdot H_{i}(\omega)
$$

In Equation (6), the FRFs and FFTs are all complex, not real. And the time domain of stress can be obtained by IFFT in Equation (7).

$$
\sigma(n)=\operatorname{real}\left(\frac{1}{N} \sum_{\mathrm{m}=0}^{N-1} \sigma(\omega) e^{j \omega m}\right)
$$

Then, the stress time history can be used to evaluate the fatigue strength or life.

\section{Stress simulation}


Firstly, the simulation finite element model of lifeguard was established by ABAQUS and modal analysis was conducted, the modal results are shown in Fig. 3, and three different directions (X, Y, Z) are defined in Fig. 3.



(a) First bending mode



(b) Second bending mode

Fig. 3. The first two modes of lifeguard

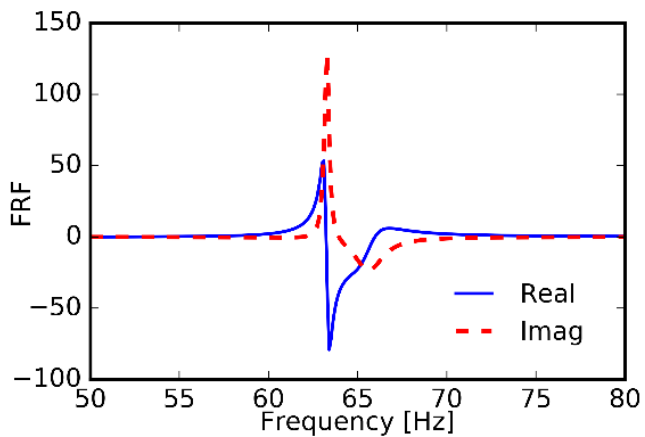

(a) X directional loading FRF



(b) Y directional loading FRF

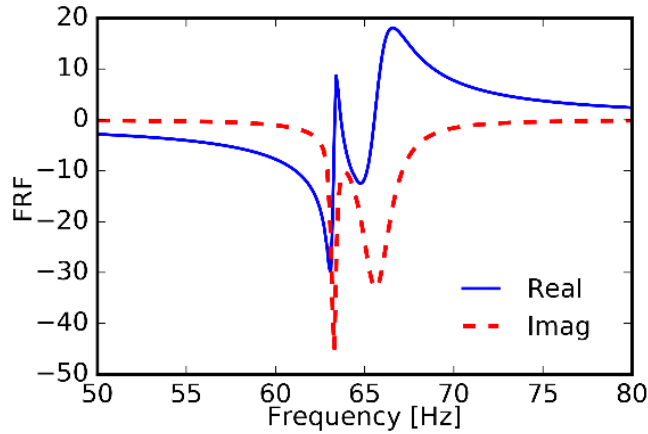

(c) $\mathrm{Z}$ directional loading FRF

Fig. 4. FRFS of three external loadings

After the modal analysis, the cracking position acceleration-stress FRFs of three different directional loadings were calculated, and the modal damper ratio of first two modes is $0.23 \%$ and $0.61 \%$ respectively. And the three FRFs are shown in Fig. 4.

\subsection{PSD stress simulation method}

Firstly, we just use the single load condition stress simulation method, the stress PSD could be calculated by Equation (8). We just call it "PSD method1".

$$
\begin{aligned}
& G(f)=\left|\mathrm{H}_{x}(f)\right|^{2} \cdot \mathrm{A}_{x}(f)+\left|\mathrm{H}_{y}(f)\right|^{2} \cdot \mathrm{A}_{y}(f) \\
& +\left|\mathrm{H}_{z}(f)\right|^{2} \cdot \mathrm{A}_{z}(f)
\end{aligned}
$$

Secondly, the Equation (4) was used to calculate the stress PSD, which is "PSD method2". The simulation results are shown in Fig. 5, which are compared to the test stress result.

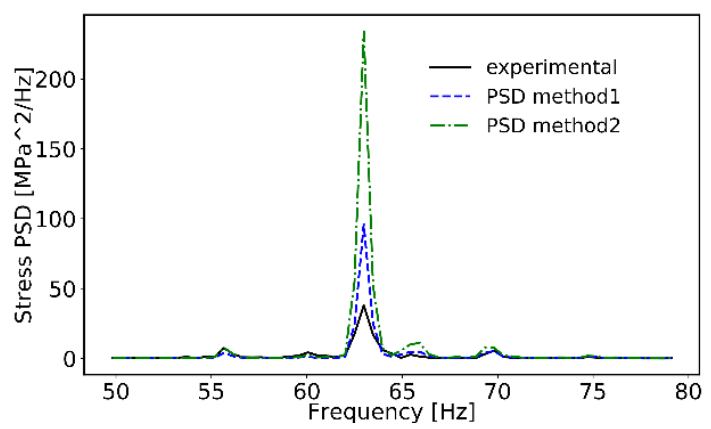

Fig. 5. Stress comparison of test and simulation by PSD

From the comparison result, we can know that the PSD stress simulation method is conservative. The reason maybe is that the PSD method just consider the different external loadings reach the maximum amplitude simultaneously. Actually, every two external loadings have the phase difference.

\subsection{FFT stress simulation method}

In this section, the FFT stress simulation method will be illustrated. First, the FFTs of external loadings are calculated, and the phase should be retained. Then, the Equation (6) will be adopted. We could get the FFT of 
dynamic stress calculated, which is used to compare with the FFT result of experimental stress in Fig. 6.

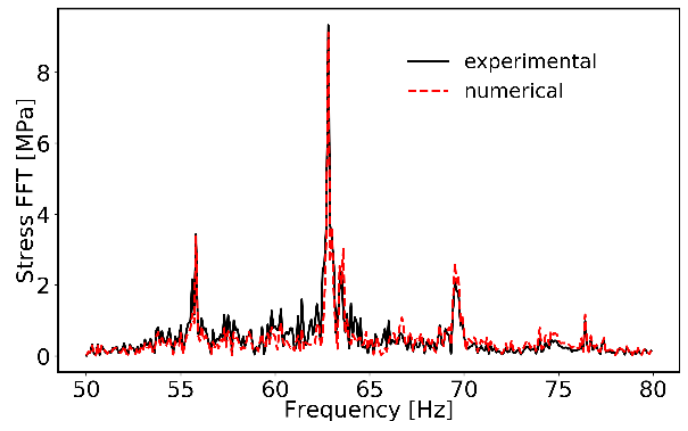

Fig. 6. Stress comparison of test and simulation by FFT

It can be seen from the Fig. 6 that the numerical results are almost same with the experimental result. The time history of stress could be calculated by IFFT according to Equation (7). Which could be seen in Fig. 7.

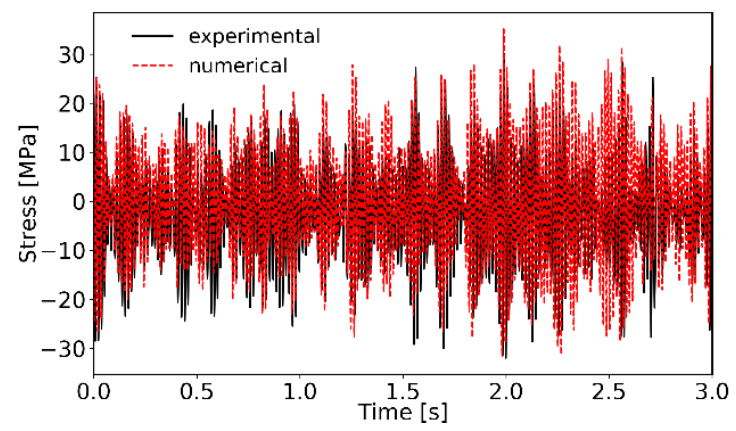

Fig. 7. Stress comparison of test and simulation by FFT

The stress PSD from three simulation methods are shown in Fig. 8. It can be seen from Fig. 8 that under multi-load condition, the dynamic stress simulation method in this paper is accurate and acceptable, however, the PSD method is conservative, especially PSD method 2.

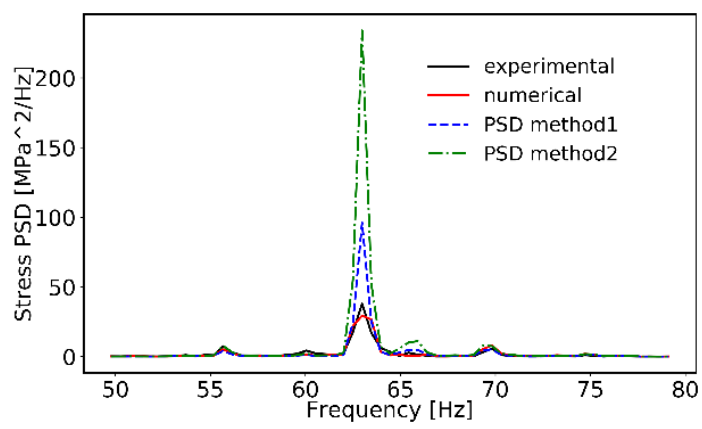

Fig. 8. Stress comparison of three methods

\section{Conclusion}

The simulation formula of dynamic stress under multiload input condition was deduced, which was based on the structure dynamics mechanics. The formula deduced in this paper was used to calculate the dynamic stress structure in resonance state, which was compared with the conventional PSD method. It was found that when use the PSD method to simulate the stress under multiload condition, the simulated result is conservative, however, the FFT method has the advantages in solving the phase information under this condition. In conventional method, the phase information is neglected when calculating the PSD of data, so, it will not predict the dynamic stress accurately. Therefore, the method in this paper is recommended, and which was verified by experimental result.

The authors gratefully acknowledge the financial support of Traction Power State Key Laboratory Research Project (Grant No. 2015TPL_Z03), Science and technology support program of China (Grant No. 2015BAG13B01-03) and Shandong Science and Technology Special Project (Grant No. 2015ZDXX0102A01)

\section{References}

1. M. Haiba, D.C. Barton, P.C. Brooks, M.C. The development of an optimisation algorithm based on fatigue life. Int J Fatigue 25, 4 (2003)

2. P. Heyes, J. Dakin, St.John C, The Assessment and Use of Linear Static FE Stress Analyses for Durability Calculations, SAE Technical Paper 951101 (1995).

3. Haiba M, Barton DC, Brooks PC, Levesley MC, Review of life assessment techniques applied to dynamically loaded automotive components, Comput Struct 80,5 (2002).

4. Moon SI, Cho IJ, Yoon D, Fatigue life evaluation of mechanical components using vibration fatigue analysis technique, J Mech Sci Technol 25, 3 (2011).

5. Yam LY, Leung TP, Li DB, Xue KZ, Theoretical and experimental study of modal strain analysis, J Sound Vib 191, 2 (1996).

6. A. Halfpenny. A frequency domain approach for fatigue life estimation from finite element analysis. Key Engineering Materials 167 (1999). 